\title{
Class I Methanol Masers in the Galactic Center
}

\author{
Loránt O. Sjouwerman ${ }^{1} \&$ Ylva M. Pihlström ${ }^{2} \dagger$ \\ ${ }^{1}$ National Radio Astronomy Observatory, P.O. Box O, 1003 Lopezville Rd., Socorro, NM 87801 \\ ${ }^{2}$ Department of Physics and Astronomy, University of New Mexico, MSC07 4220, \\ Albuquerque, NM 87131
}

\begin{abstract}
We report on 36 and $44 \mathrm{GHz}$ Class I methanol $\left(\mathrm{CH}_{3} \mathrm{OH}\right)$ maser emission in the Sagittarius A (Sgr A) region with the Expanded Very Large Array (EVLA). At least three different maser transitions tracing shocked regions in the $\mathrm{cm}$-wave radio regime can be found in Sgr A. $44 \mathrm{GHz}$ masers correlate with the positions and velocities of $36 \mathrm{GHz} \mathrm{CH}_{3} \mathrm{OH}$ masers, but the methanol masers correlate less with $1720 \mathrm{MHz} \mathrm{OH}$ masers. Our results agree with theoretical predictions that the densities and temperatures conducive for $1720 \mathrm{MHz} \mathrm{OH}$ masers may also produce 36 and $44 \mathrm{GHz} \mathrm{CH}_{3} \mathrm{OH}$ maser emission. However, many $44 \mathrm{GHz}$ masers do not overlap with $36 \mathrm{GHz}$ methanol masers, suggesting that $44 \mathrm{GHz}$ masers also arise in regions too hot and too dense for $36 \mathrm{GHz}$ masers to form. This agrees with the non-detection of $1720 \mathrm{MHz} \mathrm{OH}$ masers in the same area, which are thought to be excited under cooler or denser conditions. We speculate that the geometry of the bright $36 \mathrm{GHz}$ masers in Sgr A East outlines the location of a SNR shock front.
\end{abstract}

Keywords. Galaxy: center - Masers - Shock waves - Supernovae: individual(Sgr A East)

\section{Introduction}

The Sagittarius A complex is one of the best studied regions in the sky and encompasses several interesting phenomena like the nearest supermassive nuclear black hole (Sgr A*), the circumnuclear disk (CND), star forming regions (SFRs) and supernova remnants (SNRs). The line of sight toward the Sgr A complex consists of the SNR Sgr A East in the back and the CND (whose ionized part is known as Sgr A West) in the front partly overlapping with Sgr A East. Molecular gas is abundant and well distributed; the CND consists of irregularly distributed clumps of molecular gas and there are two giant molecular cloud cores (GMCs) called the +20 and $+50 \mathrm{~km} \mathrm{~s}^{-1}$ clouds $(\mathrm{M}-0.13-0.08$ and M-0.02 - 0.07 respectively). These GMCs form the molecular belt stretching across the Sgr A complex, providing the interstellar medium (ISM) that interacts with Sgr A East. A nice and recent comprehensive overview is presented by Amo-Baladrón, Martín-Pintado \& Martín (2011).

Bright maser lines are useful probes of physical conditions within molecular clouds, especially when mapped in detail by interferometers. One example is the collisionally pumped $1720 \mathrm{MHz} \mathrm{OH}$ maser which is widely recognized as a tracer for shocked regions, observed both in SFRs and SNRs. In SNRs, Very Large Array (VLA) observations have shown that the $\mathrm{OH}$ masers originate in regions where the SNR shock collides with the interstellar medium (e.g. Claussen et al. 1997, Yusef-Zadeh et al. 2003, Frail \& Mitchell 1998). Such OH masers are numerous in Sgr A East, thus probing the conditions of the interaction regions between the +50 and $+20 \mathrm{~km} \mathrm{~s}^{-1}$ clouds and the SNR Sgr A East.

Dense gas structures in the Galactic center region, including Sgr A East, are traced by ammonia and methanol thermal emission (Coil \& Ho 2000, Szczepanski et al. 1989,

$\dagger$ Ylva Pihlström is also Adjunct Astronomer at the National Radio Astronomy Observatory 
Szczepanski, Ho \& Gusten 1991). Methanol abundances are high enough to produce maser emission. Like $1720 \mathrm{MHz} \mathrm{OH}$ masers, Class I methanol masers such as the 36 and $44 \mathrm{GHz}$ transitions are excited through collisions. Theoretical modeling of collisional $\mathrm{OH}$ excitation predicts that the $1720 \mathrm{MHz} \mathrm{OH}$ should be found in regions of $n \geqslant 10^{5} \mathrm{~cm}^{-3}$, $T \sim 75$ K (Gray, Doel \& Field 1991, Gray, Field \& Doel 1992, Wardle 1999, Lockett, Gauthier \& Elitzur 1999, Pihlström et al. 2008). The number density and temperature required for $36 \mathrm{GHz}$ methanol masers are near those modeled for $1720 \mathrm{MHz} \mathrm{OH}$ masers, with $n \sim 10^{4}-10^{5} \mathrm{~cm}^{-3}$ and $T<100 \mathrm{~K}$ (Morimoto, Kanzawa \& Ohishi 1985, Cragg et al. 1992, Liechti \& Wilson 1996). At least in SFRs, higher densities and temperatures, $n \sim 10^{5}-10^{6} \mathrm{~cm}^{-3}$ and $T=80-200 \mathrm{~K}$, the Class I $44 \mathrm{GHz}$ line will have optimized maser output, while the $36 \mathrm{GHz}$ maser eventually becomes quenched (Pratap et al. 2008, Sobolev et al. 2005, Sobolev et al. 2007). These methanol masers may therefore constrain the density in the shocked SNR regions. In turn upper limits can be used to estimate the importance of compression by shocks in the formation of stars near SNRs.

That Class I methanol maser lines are detectable in SNR/cloud interaction regions was shown by Sjouwerman, Pihlström \& Fish (2010), using the 7 first antennas outfitted with $36 \mathrm{GHz}$ receivers at the Expanded VLA (EVLA). Several bright masers were found near the $1720 \mathrm{MHz} \mathrm{OH}$ masers in the Sgr A East molecular cloud - SNR interaction region; a feature also observed by many others (e.g. Tsuboi, Miyazaki \& Okumura 2009). To see whether the relation between the collisionally excited 36 and 44 methanol masers and $1720 \mathrm{MHz} \mathrm{OH}$ holds in general, we here present the result of a search for Class I $44 \mathrm{GHz}$ methanol maser emission in the Sgr A region.

Meanwhile we have surveyed the entire $\sim 6^{\prime} \times 8^{\prime}$ Sgr A complex for 36 and $44 \mathrm{GHz}$ methanol emission for which the complete results will be reported elsewhere; here we concentrate on the early detections in the $\sim 4^{\prime} \times 4^{\prime}$ Sgr A region.

\section{Observations and Results}

The EVLA was used in its $\mathrm{C}$ configuration to observe the transition of $\mathrm{CH}_{3} \mathrm{OH}$ at $44069.41 \mathrm{MHz}$ with a bandwidth of $8 \mathrm{MHz}$ in dual polarization at a velocity resolution of approximately $0.2 \mathrm{~km} \mathrm{~s}^{-1}$. The primary beam at $44 \mathrm{GHz}$ is about $56^{\prime \prime}$; initially we selected five pointing positions ("A" through "E", see Figure 1) with central velocities based on previous results on $1720 \mathrm{MHz} \mathrm{OH}$ masers and $36 \mathrm{GHz}$ methanol masers. Later we covered the whole Sgr A region as part of a larger $\sim 6^{\prime} \times 8^{\prime}$ survey. Position A corresponds to a region of high-velocity $1720 \mathrm{MHz}$ OH masers belonging to the circumnuclear disk, covering LSR velocities between 106 and $157 \mathrm{~km} \mathrm{~s}^{-1}$. In position B we previously detected $36 \mathrm{GHz}$ methanol masers at velocities around $23 \mathrm{~km} \mathrm{~s}^{-1}$ (LSR coverage -3 to $+39 \mathrm{~km} \mathrm{~s}^{-1}$ ). This pointing position partly overlaps on the sky with pointing position $\mathrm{C}$ which has a central velocity of $48 \mathrm{~km} \mathrm{~s}^{-1}$ (LSR coverage $22-74 \mathrm{~km} \mathrm{~s}^{-1}$ ) based on the $1720 \mathrm{MHz} \mathrm{OH}$ masers. Finally, positions D and E correspond to a region where the $50 \mathrm{~km} \mathrm{~s}^{-1}$ molecular cloud interacts with Sgr A East, and where $1720 \mathrm{MHz}$ masers are numerous. The LSR velocity coverage for these pointing positions was $22-74 \mathrm{~km} \mathrm{~s}^{-1}$.

The data were reduced using standard procedures in AIPS, using 3C 286 as the flux density calibrator resulting in a typical absolute flux density uncertainty of $15 \%$. In the fields were masers were found, a strong maser channel was used for self-calibration. Each cube was CLEANed with robust weighting down to a level of five times the theoretical rms over a field approximately twice the primary beam. This was necessary since some masers appeared in the sidelobes and needed to be accounted for in the CLEANing process. The resulting typical channel rms noise is $15-20 \mathrm{mJy} / \mathrm{beam}$, and the restoring beam is $1.3 \times 0.5^{\prime \prime}$. 
The image cubes were searched for masers, and parameters for the individual features were extracted using the AIPS task JMFIT, with peak fluxes corrected for primary beam attenuation using PBCOR. A few weaker masers exist in the cubes, but they are all located close to the brighter masers in position and velocity, and will not change any of the discussion in Sect. 3 .

A few features show more than one spectral peak at a given position, implying there is structure on scales smaller than the EVLA beam. Some spectral features have wings of weaker emission extending over $6-8 \mathrm{~km} \mathrm{~s}^{-1}$. Since the observations were taken when the EVLA was in C-configuration, we suspect that some of this broad and weak emission is of thermal origin. The peak flux density of the spectral features corresponds to brightness temperatures exceeding $10^{3} \mathrm{~K}$, but as both the $44 \mathrm{GHz}$ and $36 \mathrm{GHz}$ masers are thought to be excited by the same process, it is likely that at least the $44 \mathrm{GHz}$ detections co-located with $36 \mathrm{GHz}$ masers are masers too.
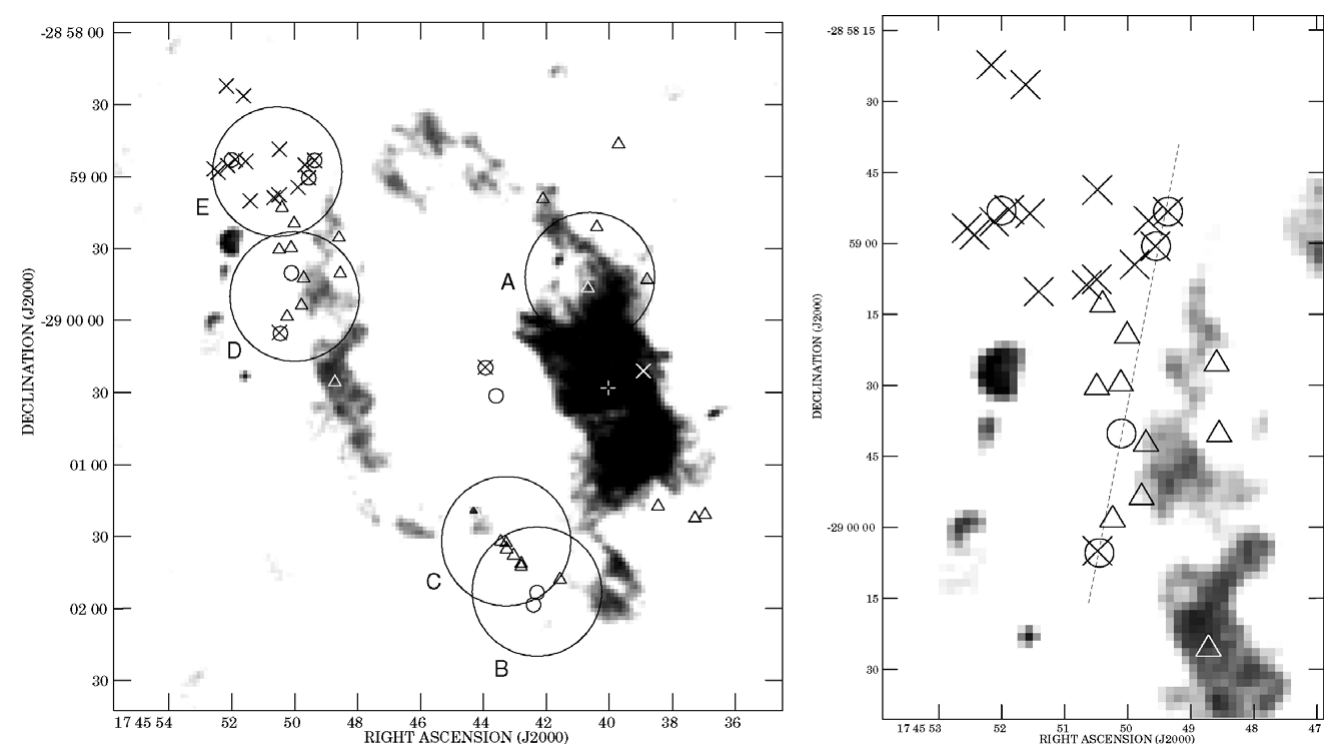

Figure 1. Relative sky positions of the $44 \mathrm{GHz}$ methanol masers (crosses), $36 \mathrm{GHz}$ methanol masers (circles) and $1720 \mathrm{MHz} \mathrm{OH}$ masers (triangles). The white plus symbol marks the position of Sgr A*. The large circles show the five field-of-view positions covered. A blow-up of the upper left region is shown at the right hand side, showing an apparent systematic offset in the location of the three maser species, with the $44 \mathrm{GHz}$ methanol masers concentrated to the northeast (upper left), the $1720 \mathrm{MHz} \mathrm{OH}$ masers more to the southwest (lower right) and a NNW-SSE line of (four) very bright $36 \mathrm{GHz}$ methanol masers, three co-spatial with $44 \mathrm{GHz}$, roughly dividing the two regions. The dashed line shows the alignment of $36 \mathrm{GHz}$ masers with the shock front in the northnorthwest-southsoutheast direction.

Three masers were detected outside the primary beam (large circles in Figure 1) and their flux density is therefore less certain than for masers located within the primary beam. The position and velocity of maser 2 at $50 \mathrm{~km} \mathrm{~s}^{-1}$ agrees very well with the position of a $36 \mathrm{GHz}$ maser at $51 \mathrm{~km} \mathrm{~s}^{-1}$ (Sjouwerman, Pihlström \& Fish 2010), and we therefore trust that this detection is real. This $44 \mathrm{GHz}$ maser has previously been reported on by Yusef-Zadeh et al. (2008), and is associated with a molecular clump 'G'. Similarly, we confirm their maser ' $V$ ' (our maser 1) in our data taken for the phasereference calibrator J1745-2900 (i.e. Sgr A*), and both 'G' and 'V' in archival VLA data taken on 2009 April 23. The $44 \mathrm{GHz}$ maser associated with clump 'F' reported by YusefZadeh et al. (2008) is not confirmed by our, nor the archival, observations. The additional 
two masers detected outside the primary beam also do not have $36 \mathrm{GHz}$ masers directly associated with them.

Figure 1 plots the position of the detected $44 \mathrm{GHz}$ methanol sources compared to the previously detected $36 \mathrm{GHz}$ and $1720 \mathrm{MHz} \mathrm{OH}$ masers. The right hand side panel of Figure 1 shows the northeast region of Sgr A East where most $44 \mathrm{GHz}$ masers are located. From these plots a few main results can be concluded. Firstly, the positions and velocities of several $44 \mathrm{GHz}$ masers (Figure 1) agree to within the errors with the values reported by Sjouwerman, Pihlström \& Fish (2010) for the $36 \mathrm{GHz}$ masers. Secondly, there is a systematic difference between the overall distributions of the $1720 \mathrm{MHz} \mathrm{OH}, 36 \mathrm{GHz}$ methanol and $44 \mathrm{GHz}$ methanol masers. In the northeast (upper left) region, in overlap with the densest part of the $50 \mathrm{~km} \mathrm{~s}^{-1}$ cloud, the $44 \mathrm{GHz}$ masers are offset to the northeast with respect to a narrow, almost linear southsoutheast to northnorthwest distribution of the $36 \mathrm{GHz}$ masers. The $1720 \mathrm{MHz} \mathrm{OH}$ masers are found on the other side of the $36 \mathrm{GHz}$ masers, near the radio continuum of the SNR to the southwest (lower right). A positional offset between the $\mathrm{OH}$ and methanol is also observed in the southeastern interaction region, where the SNR G359.02-0.09 overlaps the Sgr A East continuum (Coil \& Ho 2000, Herrnstein \& Ho 2005). These positional offsets are discussed further in Sect. 3.

\section{Discussion}

\section{1. $36 \mathrm{GHz}$ versus $44 \mathrm{GHz}$ Methanol Masers}

Modeling of methanol masers suggest that the $36 \mathrm{GHz}$ transition occurs under somewhat cooler and less dense $\left(T \sim 30-100 \mathrm{~K}, n \sim 10^{4}-10^{5} \mathrm{~cm}^{-3}\right)$ conditions than the $44 \mathrm{GHz}$ transition $\left(T \sim 80-200 \mathrm{~K}, n \sim 10^{5}-10^{6} \mathrm{~cm}^{-3}\right.$; see, e.g. Pratap et al. 2008). The range of physical conditions do however overlap, and some spatial overlap could therefore be expected. Seven $44 \mathrm{GHz}$ masers show an almost perfect overlap in both position and velocity with $36 \mathrm{GHz}$ masers (Sjouwerman, Pihlström \& Fish 2010). Here, according to the modeling, the densities and temperatures should be close to $10^{5} \mathrm{~cm}^{-3}$ and $100 \mathrm{~K}$ to produce both methanol maser lines.

It is striking that the brightest $36 \mathrm{GHz}$ masers, all to the northwest in positions $\mathrm{D}$ and E (Sjouwerman, Pihlström \& Fish 2010), are narrowly distributed along a line roughly from north to south, of which three coincide with $44 \mathrm{GHz}$ masers in position and velocity within the errors. This NNW-SSE division more or less coincides with the sharp gradient in low-frequency radio continuum emission of the Sgr A East SNR (Pedlar et al. 1989) and appears to be located in the sheath in the CS emission as mapped by Tsuboi, Miyazaki \& Okumura (2009). The mean velocity of each transition is $46 \mathrm{~km} \mathrm{~s}^{-1}$, implying that they arise in similar regions of the molecular cloud where the velocities still are less disturbed by the SNR shock (see Sect. 3.2). Apart from two individual exceptions located far from this area, we do not find any $44 \mathrm{GHz}$ (nor $36 \mathrm{GHz}$ ) masers westward of this line in our pointings. It is therefore tempting to speculate that the line delineates the arrival of the shock front, where enough material has been swept up to provide the density for the creation of (perhaps due to geometry very bright $36 \mathrm{GHz}$ ) methanol masers, but not yet enough energy has dissipated to dissociate all methanol or to significantly disturb the velocity structure by means of a reverse shock (Section 3.2).

In the northeastern part of Sgr A East toward the core of the $50 \mathrm{~km} \mathrm{~s}^{-1}$ cloud, there is a group of $44 \mathrm{GHz}$ masers with a distinct positional offset from the $36 \mathrm{GHz}$ masers. The narrower distribution of $36 \mathrm{GHz}$ masers suggests that the conditions required to produce masers in this transition are not fulfilled further to the northeast. The position of the $36 \mathrm{GHz}$ emission is consistent with being just in the $\mathrm{SNR} /$ cloud interaction region, while 
the $44 \mathrm{GHz}$ masers may be found deeper inside the denser parts of the cloud. These $44 \mathrm{GHz}$ masers, which are typically found to be brighter than the $36 \mathrm{GHz}$ masers, may originate near sites of massive star formation instead (e.g. Pratap et al. 2008, Fish et al. 2011). The lack of companion $36 \mathrm{GHz}$ masers in this putative star-forming region (e.g. Tsuboi, Miyazaki \& Okumura 2009) therefore may be due to the limited sensitivity of the $36 \mathrm{GHz}$ observations. This picture, at least for this region in the Galactic center, in which $44 \mathrm{GHz}$ masers are primarily associated with cloud cores and $36 \mathrm{GHz}$ masers are found at the boundaries of the SNR interaction regions, is consistent with theoretical models indicating that $44 \mathrm{GHz}$ masers can be produced at higher densities than $36 \mathrm{GHz}$ masers. The existence of $36 \mathrm{GHz}$ masers without accompanying $44 \mathrm{GHz}$ masers in positions $\mathrm{B}$ and $\mathrm{C}$ may then indicate the interaction region of two SNRs without the presence of a dense cloud.

\subsection{OH versus Methanol Masers}

As is the case with Class I methanol masers, $1720 \mathrm{MHz} O \mathrm{OH}$ masers are used as tracers of shocked regions. The presence of $1720 \mathrm{MHz}$ OH masers indicates the presence of C-shocks (e.g. Lockett, Gauthier \& Elitzur 1999). Modeling of $\mathrm{OH}$ and $\mathrm{CH}_{3} \mathrm{OH}$ shows that the three maser transitions discussed here require similar densities and temperatures. This agrees well with the detection of all three masers in Sgr A East. However, we observe a distinct offset in positions between the methanol and $\mathrm{OH}$ masers (Figure 1). In the northeast interaction region between the $50 \mathrm{~km} \mathrm{~s}^{-1}$ cloud and $\mathrm{Sgr}$ A East the $\mathrm{OH}$ masers are found more to the southwest. In addition, the $1720 \mathrm{MHz} O H$ masers have a higher mean velocity of $\sim 57 \mathrm{~km} \mathrm{~s}^{-1}$ versus $\sim 46 \mathrm{~km} \mathrm{~s}^{-1}$ for the methanol. However, the $1720 \mathrm{MHz}$ $\mathrm{OH}$ does overlap in the sky with the line of $36 \mathrm{GHz}$ masers.

A similar offset is observed in the southeastern interaction region in pointing positions $\mathrm{B}$ and $\mathrm{C}$, where the methanol masers are offset southwest from the $\mathrm{OH}$. The $\mathrm{OH}$ mean velocities here are $58 \mathrm{~km} \mathrm{~s}^{-1}$ to be compared to the $24.5 \mathrm{~km} \mathrm{~s}^{-1}$ for the $36 \mathrm{GHz}$ methanol. No $44 \mathrm{GHz}$ methanol was detected in this region.

The association between $1720 \mathrm{MHz} \mathrm{OH}$ and $36 \mathrm{GHz}$ methanol emission may be due to the processes that form these molecules. $\mathrm{OH}$ is created by dissociation of $\mathrm{H}_{2} \mathrm{O}$ (and maybe also $\mathrm{CH}_{3} \mathrm{OH}$ ), and the propagation of a $\mathrm{C}$-shock creates densities and temperatures suitable for $1720 \mathrm{MHz} \mathrm{OH}$ inversion. Thus, OH masers should preferentially be found in the SNR post-shock region. This agrees with the $\mathrm{OH}$ masers being co-located with positions of radio continuum, outlining regions where electrons have been accelerated by the shock. The production of methanol is less well understood, but it is believed that methanol is released from grains, either by sputtering from a shock or by evaporation when temperatures reach above $100 \mathrm{~K}$ (Hidaka et al. 2004, Menten et al. 2009, Bachiller \& Perez Gutierrez 1997, Voronkov et al. 2006, Hartquist et al. 1995).

\section{References}

Amo-Baladrón, M. A., Martín-Pintado, J., \& Martín, S. 2011, A\&AA, 526, A54

Bachiller, R. \& Perez Gutierrez, M., 1997, ApJ, 487 L93

Claussen, M. J., Frail, D. A., Goss, W. M., \& Gaume, R. A. 1997, ApJ, 489, 143

Coil, A. L. \& Ho, P. T. P. 2000, ApJ, 533, 245

Cragg, D. M., Johns, K. P., Godfrey, P. D., \& Brown, R. D., 1992, MNRAS, 259, 203

Fish, V. L., Muehlbrad, T. C., Pratap, P., Sjouwerman, L. O., Strelnitski, V., Pihlström, Y. M., \& Bourke, T. L., 2011, ApJ, 729, 14

Frail, D. A. \& Mitchell, G. F. 1998, ApJ, 508, 690

Gray, M. D., Doel, R. C., \& Field, D. 1991, MNRAS, 262, 30

Gray, M. D., Field, D., \& Doel, R. C. 1992, A\&AA, 264, 220 
Hartquist, T. W., Menten, K. M., Lepp, S., \& Dalgarno, A., 1995, MNRAS, 272, 184

Herrnstein, R. M. \& Ho, P. T. P. 2005, ApJ, 620, 287

Hidaka, H., Watanabe, N., Shiraki, T. m Nagaoka, A., \& Kouchi, A., 2004, ApJ, 614, 1124

Liechti, S. \& Wilson, T. L., 1996, A\&SA, 314, 615

Lockett, P., Gauthier, E., \& Elitzur, M. 1999, ApJ (Letters), 511, L235

Menten, K. M., Wilson, R. W., Leurini, S., \& Schilke, P., 2009, ApJ, 692, 47

Morimoto, M., Kanzawa, T., \& Ohishi, M., 1985, ApJ (Letters), 288, L11

Pedlar, A., Anantharamaiah, K. R., Ekers, R. D., Goss, W. M., van Gorkom, J. H., Schwarz, U. J., \& Zhao, J.-H. 1989, ApJ, 342, 769

Pihlström, Y. M., Fish, V. L., Sjouwerman, L. O., Zschaechner, L. K., Lockett, P. B., \& Elitzur, M., ApJ, 676, 371

Pratap, P., Shute, P. A., Keane, T. C., Battersby, C., \& Sterling, S., 2008, AJ, 135, 1718

Szczepanski, J. C., Ho, P. T. P., Haschick, A. D., \& Baan, W. A., 1989, IAU Symp. 136, 383

Szczepanski, J. C., Ho, P. T. P., \& Gusten, R. 1991, ASP Conf. Series, Vol. 16, 143

Sjouwerman, L. O., Pihlström, Y. M., \& Fish, V. L. 2010, ApJ (Letters), 710, L111

Sobolev, A. M., Cragg, D. M., Ellingsen, S. P., Gaylard, M. J., Goedhart, S., Henkel, C., Kirsanova, M. S., Ostrovskii, A. B., Pankratova, N. V., Shelemei, O. V., van der Walt, D. J., Vasyunina, T. S., \& Voronkov, M. A., 2007, IAU Symp., 242, Vol. 242, 81

Sobolev, A. M., Ostrovskii, A. B., Kirsanova, M. S., Shelemei, O. V., Voronkov, M. A., \& Malyshev, A. V., 2005, IAU Symp. 227, Vol. 227, 174

Tsuboi, M., Miyazaki, A., \& Okumura, S. K. 2009, PASJ, 61, 29

Voronkov, M. A., Brooks, K. J., Sobolev, A. M., Ellingsen, S. P., Ostrovskii, A. B., \& Caswell, J. L., 2006, MNRAS, 373, 411

Wardle, M. 1999, ApJ (Letters), 525, L101

Yusef-Zadeh, F., Braatz, J., Wardle, M., \& Roberts, D. 2008, ApJ (Letters), 683, L147

Yusef-Zadeh, F., Wardle, M., Rho, J., \& Sakano, M. 2003, ApJ, 585, 319 